Mr. H. Littlewood, Central Council of Physical Recreation

There were certain underlying principles that Carlisle had outlined - the training should be severe - individuals react differently to the same training - exercise was only one of a number of stresses - recovery perinds both during training sessions and throughout the year were necessary. Carlisle also maintained that training should be specific, although there was a certain amount of carryover the best training for a particular sport is not necessarily the best for another sport. Strengthening and flexibility exercises are beneficial and should preferably be carried out in the off season. Interval training is perhaps the most important individual training item. Nutrition plays a significant part in any physical performance.

The above principles were followed to a greater or lesser extent in the sports that had been studied. Lawn tennis players carried out circuit training, were encouraged to run in open country and had a series of specific training games.

Long distance skiers, according to the Observer, found that lumber jacking was an ideal means of training, the Swedes did gymnastics and ran for long periods over difficult terrain. The skiers faced staleness, and relaxation was an important part of the training, as was diet. Three-quarters of the training time was spent off the snow.

Swimmers had two main aspects of training - land conditioning and training in the water. Land conditioning was concerned with the general level of fitness and included weight training, the use of pulleys, expanders and isometric muscle contractions for strength, circuit training and fartlek running for endurance and special exercises for mobility. The training in the water was based on the theory that repeated speed swims over a short distance were better than longer swims over a greater distance. The controlled interval method of training, where the swimmer carried out repeated fast swims with a definite recovery interval, was extensively practised.

In Association Football the method of training for professional players had changed considerably. Attention was now paid to very hard training sessions with the ball, to running specific distances and to mobility and agility exercises. Much of the training with the ball was done in small groups, the practices carried out having a direct relationship to the game as a whole. These practices were often very intensive and were followed, as a means of recovery, by other activities of a less strenuous nature. 
Weight lifters found lack of time for training a very serious handicap. The training of an international weight lifter laid great emphasis on performing a series of low repetitions with very heavy weights.

\section{Problems in Training Oarsmen - Staleness}

Dr. J. R. Owen, Team Manager, Amateur Rowing Assn.

Staleness is fairly common in rowing and is probably more common than is recognised or admitted in other sports as well. As a condition it is psychological in origin with physical manifestations.

When fully developed it may be recognised by lethargy, lack of interest, tiredness and lack of will or even desire to win. At this stage it is too late to do anything about it. Staleness may manifest itself suddenly when a crew or individual fails to reproduce in competition the form shown in training and practice.

The signs of impending staleness most commonly recognised are:-

1. Undue tiredness, out of all proportion to the exercise taken,

2. Insomnia,

3. Lack of interest, not inly in the sport itself but in outside interests, and

4. Lack of confidence in the ability to win.

Other less obvious signs include:-

5. An abnormal anxiety about the chances,

6. Slight and increasing irritability about unimportant things,

7. Complaints of various trifling ailments, and

8. Alteration in weight (usually slight progressive loss) unrelated to diet and exercise taken.

Treatment consists in prevention - this is the only thing that is effective. The early signs must be recognised and dealt with, and the causes appreciated and prevented from arising.

It must always be clearly shown that the condition is not physical and the training programme reviewed and the individual(s) checked to see that no illness is developing or infection being incubated. 